\title{
A study of quasi reversible nitro radical anion from $\beta$-nitrostyrene at wax-impregnated carbon paste electrode
}

\author{
RONALD J MASCARENHAS, ${ }^{1}$ IRISHI N NAMBOOTHIRI, ${ }^{2}$ B S SHERIGARA ${ }^{3, *}$ and \\ VIJAYAKUMAR K REDDY ${ }^{1}$ \\ ${ }^{1}$ Department of Chemistry, St. Joseph's College, Lalbagh Road, Bangalore 560027 \\ ${ }^{2}$ Department of Organic Chemistry, Indian Institute of Technology Bombay, Mumbai 400076 \\ ${ }^{3}$ Department of Post Graduate Studies and Research in Industrial Chemistry, Kuvempu University, \\ Shankaraghatta 577451 \\ e-mail: bssherigara@rediffmail.com
}

MS received 3 November 2005; revised 17 March 2006

\begin{abstract}
A comprehensive study of the electrochemical reduction of $\beta$-nitrostyrene and the corresponding heterocyclic analogue has been carried out in aprotic media using wax-impregnated carbon paste electrodes. Nitrostyrene exhibits quasi-reversible reduction process in aprotic medium at the waximpregnated carbon paste electrodes as compared to other electrodes reported in the literature. The nitroradical anion couple detected in the presence of tetrabutyl ammonium perchlorate is found to be stable only in aprotic media. Though, as reported, the pharmacological activity related to this nitro radical anion and its therapeutic value are related to the stability of the nitro radical anion, the stability itself depends on the electrode system employed. Added benzoic acid is found to bring about a positive shift in cathodic peak potential.
\end{abstract}

Keywords. Electrochemical reduction; $\beta$-nitrostyrene; wax-impregnated carbon paste electrode; nitro radical anion.

\section{Introduction}

Significant number of pharmologically active substances have aromatic nitro groups in their molecular structure, which accounts for their biological activity. Large numbers of nitro-derivatives are used in pharmaceuticals on account of the redox process the nitro group undergoes as it enters into living organisms. The mechanism of action is their ability to accept electrons and render the nitro group susceptible for reduction, being metabolized to the corresponding amines via the nitro radical anion, nitroso and hydroxylamine intermediates. Their therapeutic use ${ }^{1}$ is mainly due to the ease of reduction of the nitro moiety in the presence of different enzymatic systems (flavin-dependent reductase, cytochrome $\mathrm{P}_{450}$ ). The pharmacological effects are largely determined by their redox responses at different $\mathrm{pH}$ values. ${ }^{2-5}$ Telomerase activity is expressed in most types of cancer cells, suggesting that telomerase may be an important target for cancer chemotherapy. Nitrostyrene

*For correspondence derivatives are found to be potent inhibitors of human telomerase. ${ }^{6}$ The nitrostyrene moiety has been identified as the structure essential for apoptosis induction mediating the prominent pro-apoptotic effects of the Michael adduct of ascorbic acid, a new synthetic phosphatase inhibitor. ${ }^{7}$ The nitro radical anion formed in the metabolic pathway is said to exhibit cytotoxicity ${ }^{8,9}$ in several systems. Redox properties control most biological responses of nitro compounds; the formation of the one-electron nitro couple seems to be an obligatory intermediate for therapeutic selectivity towards anaerobes. ${ }^{10}$ Nitrocompounds are used in treatments such as for cancer therapy, acting as specific cytotoxins and as markers for hypoxic regions in tumours. ${ }^{11}$ Heterocyclic nitrocompounds like 5-nitrofurons and 5-nitroimidazoles are used as antibacterial, antiprotozoal and anticancer agents. ${ }^{12,13}$

Though biological electron-transfer reactions are complicated, both biological and electrochemical electron-transfer reactions have many things in common. Both involve essentially heterogeneous electron-transfer processes, are $\mathrm{pH}$ - and tempera- 
ture-dependent and occur at electrode/electrolyte or membrane/solution interfaces. Hence, explanations based on electrochemistry have played an important role in interpreting and understanding the biological phenomena.

As reported, cyclic voltammetry has proved to be an important tool to study this radical species originating from different nitro-compounds. ${ }^{14,15}$ Prompted by the above observations we carried out the electrochemical reductive investigation of these compounds in non-aqueous media in order to investigate the relationship between nitro radical anion stability and its therapeutic value.

Polarographic studies have reported four-electron reduction to form phenyl acetaldoxime followed by two-electron reduction of $-\mathrm{C}=\mathrm{N}-$ bond to form phenyl hydroxylamine. ${ }^{16}$ Steric and electronic effects ${ }^{17}$ on the electro reduction of $\beta$-nitrostyrene derivatives with a substituent at the para position of the aromatic ring and a substituent $\mathrm{C}_{\beta}$ of the side chain have been reported at the mercury electrode. In aprotic media formation of reversible nitro radical anions has been reported.

To the best of our knowledge, there is no report on the study of $\beta$-nitrostyrene derivatives using carbon paste electrodes or wax impregnated carbon paste electrodes (WICPE). The motivation for using WICPE is that there is further scope for electrode surface modification through immobilization of electrocatalytic molecules, incorporation of carbon nano particles and in situ generation of $\mathrm{Hg}$ by bulkmodifying WICPE with mercuric salts, etc. and hence to carry out further investigation. Thio-compounds are suitable modifiers for thiophilic metals by forming either the corresponding salts or complexes. Compound $\mathbf{1 b}$ is used as a prototype nitrohetroaromatic system to compare the reduction potential since reduction potential is also related to toxicity. The stability of the nitroradical anion varies moving from aromatic to hetroaromatic systems. The structures of the compounds studied are as in chart 1.<smiles>O=[N+]([O-])C=Cc1ccccc1</smiles>

1a

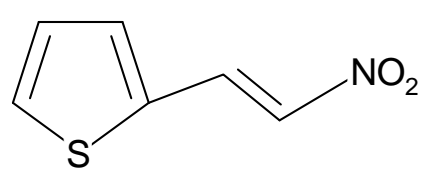

1b

\section{Experimental}

\subsection{Reagents and chemicals}

Pure [(E)-2-nitrovinyl] benzene (1a) ( $\beta$-nitrostyrene) and 2-[(E)-2-nitrovinyl] thiophene (1b) were prepared by well-known methods cited in the literature ${ }^{18,19}$ using nitromethane and aromatic/heterocyclic aldehydes. Adequate quantities of the above compounds were weighed out to prepare solutions. The final concentration of the analyte was adjusted to $2 \times 10^{-3} \mathrm{M}$. DMF used as aprotic solvent was of AR grade. Spectroscopic grade carbon powder was used for the preparation of carbon paste. Tetra-butyl ammonium perchlorate (TBAP) was used as supporting electrolyte in non-aqueous solutions.

\subsection{Instrumentation and apparatus}

The electrochemical experiments were carried out using a potentiostat provided with the Data Acquisition PC interface Card fabricated at Analytical Chemistry Division, Bhabha Atomic Research Centre, Trombay, Mumbai, compatible with an IBM PC and coupled to a printer. Experiments were performed using a one-compartment three-electrode system in which WICPE was the working electrode, saturated calomel electrode (SCE) the reference electrode and platinum the auxiliary electrode.

\subsection{Preparation of the working electrode}

Wax-impregnated carbon powder was prepared by taking $9.5 \mathrm{~g}$ of spectroscopic grade carbon powder in a quartz tube, and placing it in a tube furnace. It was then heated to a high temperature to remove adsorbed oxygen ${ }^{20}$ on the porous carbon particles. The mass was then added to an appropriate quantity of ceresin wax dissolved in warm $n$-hexane ${ }^{21}$ placed in a water bath, stirred and heated till the $n$-hexane evaporated. The wax-impregnated carbon powder was mixed with the binder, silicone oil, in the ratio $5: 3 \mathrm{w} / \mathrm{w}$. in an agate mortar and homogenized using the pestle. The electrode consisted of a teflon well, mounted at the end of a teflon tube. The prepared paste was filled into the teflon well. A copper wire fixed to a graphite rod and inserted into the teflon tube served to establish electrical contact with the external circuit. The electrode surface of the working electrode was renewed mechanically by smoothing some paste off and then polishing on a piece of

Chart 1. 
transparent paper before conducting each of the experiments. The experiments were performed in unstirred solutions.

\section{Results and discussion}

\subsection{Characteristics of reduction processes in non-aqueous medium}

Cyclic voltammograms were obtained with $2.0 \times 10^{-3} \mathrm{M}$ solutions of $1 \mathbf{a}$ and $1 \mathbf{b}$ in $0.05 \mathrm{M}$ TBAP supporting electrolytes on a DMF background. Scan rate was varied from 0.5 to $2 \mathrm{Vs}^{-1}$. The study in this medium resembles the lipophilic environment found in biological membranes. The plot of peak current against square root of scan rate in figure 1 shows linear dependence fulfilling the conditions for diffusioncontrolled processes. The plot of log of scan rate against $\log i_{p c}$ in figure 2 also shows a linear relationship $\left(R^{2}=0.9962\right)$ with a slope of 0.565 which further confirms the diffusion-controlled nature of the reduction process. The theoretical value reported in the literature for this slope is 0.5 for a diffusion peak and 1 for an adsorption peak. ${ }^{22}$ The cyclic voltammogram obtained shows an oxidation peak only at higher scan rates. There is a negative shift of the peak potential with scan rate. The cyclic voltammogram of compound $\mathbf{1 b}$ in $100 \%$ DMF at a scan rate of $1 \mathrm{Vs}^{-1}$ is shown in figure 3. This corresponds to a mono-electron reduction of the nitro-group in order to produce a nitro radical anion according to the following mechanism.

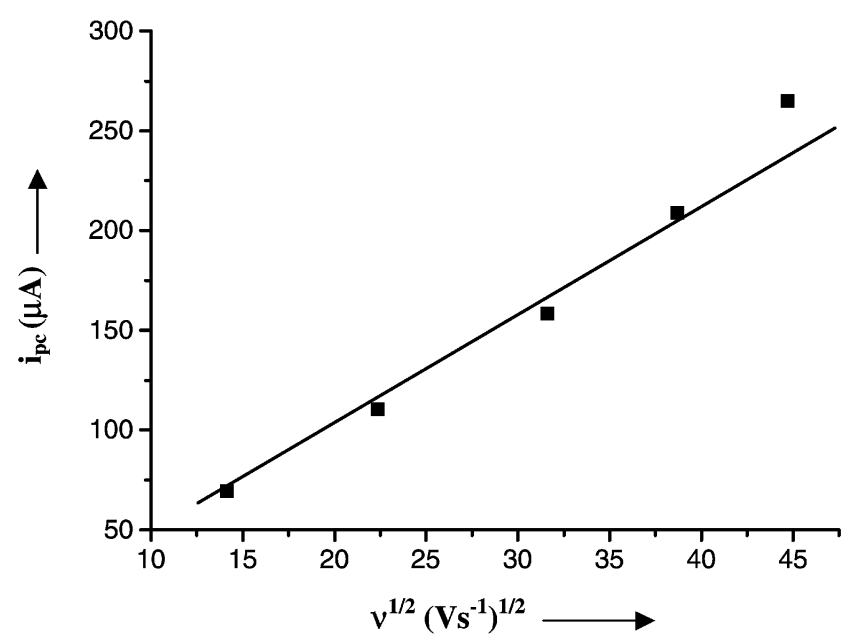

Figure 1. Effect of variation of scan rates on the peak currents of $2 \times 10^{-3} \mathrm{M}$ (compound 1a) in $0.08 \mathrm{M}$ TBAP as supporting electrolyte in DMF at WICPE.

$$
\mathrm{X}-\mathrm{CH}=\mathrm{CHNO}_{2}+e^{-} \leftrightarrows \mathrm{X}-\mathrm{CH}=\mathrm{CHNO}_{2}^{--} .
$$

The one-electron couple $\left(\mathrm{ArNO}_{2} / \mathrm{ArNO}_{2}^{-}\right)^{-}$was not observed in the mixed aqueous medium even at higher scan rates due to the instability of the nitro radical anion as it gets protonated in aqueous media. It is reported that generally the nitro radical anion is stabilized in mixed media in compounds that have pharmacological activity related to this free radical. From this it appears that derivatives of neither compound 1a nor 1b may be usable as a source of free radicals and hence would not be suitable as therapeutic agents. ${ }^{23-26}$ In our study we report that this need not be true as it depends on the type of electrode system employed. Evidently, it depends upon the rate of charge transfer and at WICPE it follows slow kinetics.

In aprotic media, the nitro-radical anion was found to be more stable. In the case of compound $\mathbf{1 b}$, peak potential shifted in the positive direction indicating less energy requirement for the formation of nitro radical anion in these compounds. Compounds with higher electroaffinity (smaller reduction potential) are reported to be more toxic. ${ }^{27}$ The $\Delta E p$ values for the couple were more than $90 \mathrm{mV}$, which increased with increase in the sweep rate. The cathodic peak had a width of $73 \pm 18 \mathrm{mV}$, which implies that the process corresponds to the reduction of the nitro compound to the nitro radical anion as given by (1). The process does not correspond to a reversible electron transfer as the ratio of $i_{p a} / i_{p c}$ is always much lower than unity. If the species formed during the

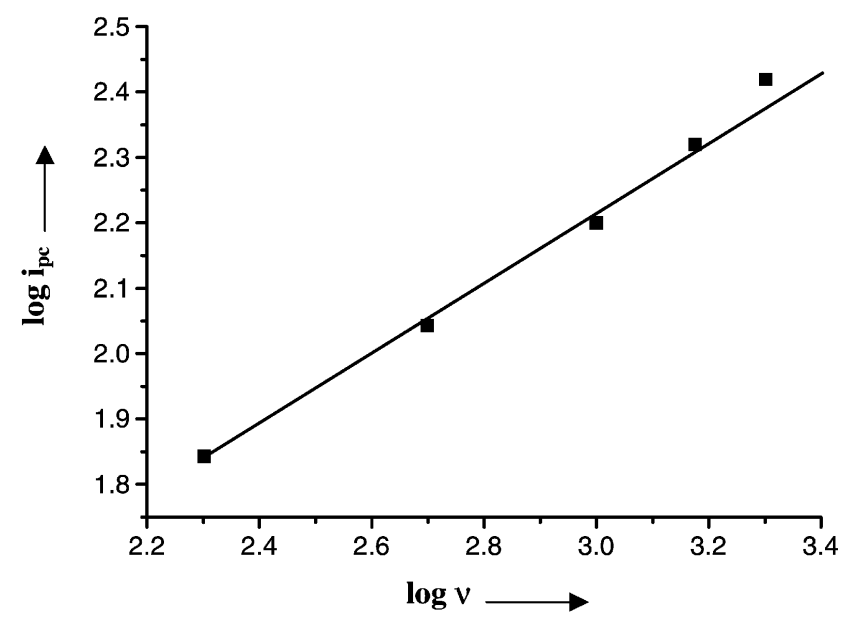

Figure 2. Plot of $\log v$ vs $\log i_{\mathrm{pc}}$ of $2 \times 10^{-3} \mathrm{M}$ (compound 1a) in $0.08 \mathrm{M}$ TBAP as supporting electrolyte in DMF at WICPE. 


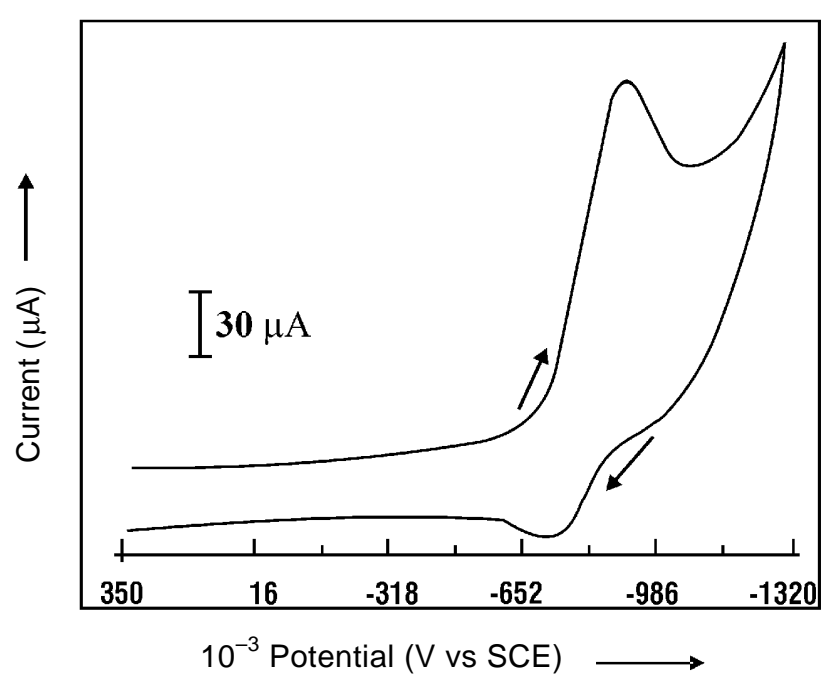

Figure 3. Voltammogram of mono electron reduction of nitrogroup of $\mathbf{1 b}$ resulting in the production of nitro radical anion in 0.05 $\mathrm{M}$ TBAP at WICPE.

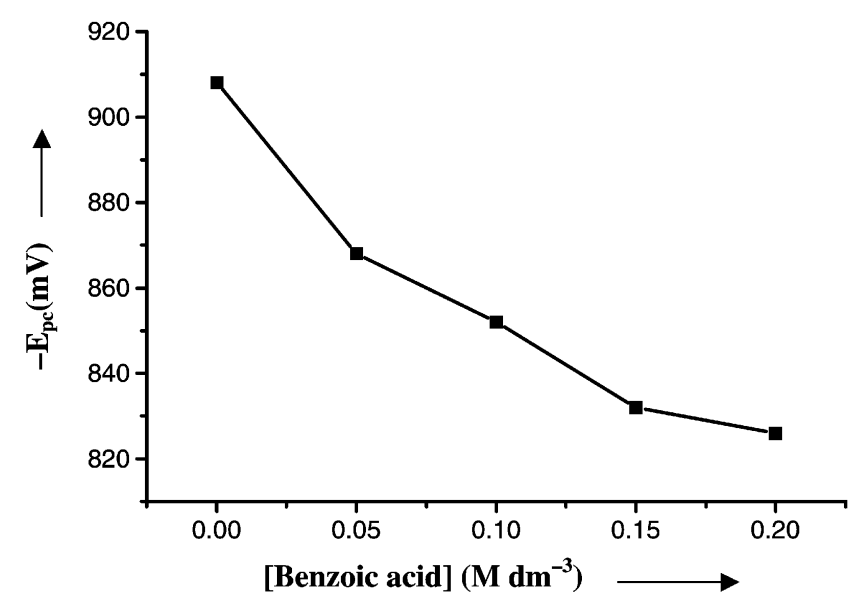

Figure 4. Plot of variation of (benzoic acid) on the peak potential of $2 \times 10^{-3} \mathrm{M}$ (compound 1a) in $0.05 \mathrm{M}$ TBAP as supporting electrolyte with a scan rate of $0.02 \mathrm{Vs}^{-1}$.

reduction is stable on the electrochemical time scale, then both forward and backward current responses are equal, giving an $i_{p a} i_{p c}$ ratio of unity.

Decrease in the concentration of the species available for reoxidation gives a ratio less than unity. This may be due to the existence of surface heterogeneous protonation. The above results observed are different as compared to other solid electrodes for such systems and do not fulfill the criteria for reversibility but correspond to a quasi-reversible nature of the electron transfer at WICPE. The presence of pasting liquid at the surface of WICPE decreases the electron transfer rate (slower kinetics), causing a higher overpotential compared to homogeneous electrodes. ${ }^{28}$ The increasing lipophility of the pasting liquid enhances the electrode overpotential. The marked hydrophobicity of the pasting liquid hinders the access of analyte towards the surface.

Generation of the nitro radical anion depends on the energy requirements to introduce one electron at the nitro moiety. ${ }^{29}$ The stability of the anion depends on the reaction media and is reflected in the decay of the corresponding oxidation current subsequent to its electrochemical generation. The current ratio equals unity in the absence of further reaction of $\mathrm{RNO}_{2}^{--}$but decreases if the radical reacts subsequently. Therefore, $\mathrm{CV}$ experiments can be used to prove the stability of the $\mathrm{RNO}_{2}^{--}$species.

\subsection{Effect of addition of benzoic acid}

Keeping the concentration of compound 1a constant at $2.0 \times 10^{-3} \mathrm{M}$ in $0.05 \mathrm{M}$ TBAP as the supporting electrolyte, the concentration of benzoic acid was increased and the voltammograms were recorded. The protonated species are more rapidly reducible and hence reduction peak shifts in the positive direction $^{30}$ due to the ionization of added benzoic acid. Figure 4 shows the effect of addition of benzoic acid on the peak potentials of compound 1a in non-aqueous medium. It is observed to shift from $-0.908 \mathrm{~V}$ to $-0.845 \mathrm{~V}$ as the concentration of benzoic acid increases from 0.05 to $0.2 \mathrm{M}$. The peak current also increases with increase in concentration of benzoic acid due to the protonation and hence electro-reduction takes place faster.

\section{Conclusions}

The nitroalkenes studied form quasi-reversible couples of nitroradical anion in aprotic medium at WICPE. The nitroradical anion in unprotonated form has greater stability. True that the pharmacological activity depends on nitro radical anion stability but the stability itself depends on the type of electrode system employed for their study. In the case of hetrocyclic derivatives, less energy is required for the formation of the nitro radical anion. Protonated species are more rapidly reducible and hence reduction peak shifts in the positive direction due to the ionization of added benzoic acid in non-aqueous medium. The redox route for the nitro group depends on the electrode system. 


\section{References}

1. Squella J A, Huerta M, Bollo S, Pessoa H and NunezVergara L J 1997 J. Electroanal. Chem. 42063

2. Sivasankar P and Reddy S J 1990 Contemporary electroanalytical chemistry (eds) L A Ivaska and R Sara (New York: Plenum)

3. Squella J A, Bollo S, La Fuente J De and NunezVergara L J 1994 Bioelectrochem. Bioenergy 3413

4. Olive P L 1979 Cancer Res. 394512

5. Wilson W R, Anderson R F and Denny W $1989 \mathrm{~J}$. Med. Chem. 3223

6. Kim J H, Lee G E, Lee J E and Chung I K 2003 Mol. Pharmacol. 631117

7. Kaap S, Quentin I, Tamiru D, Shaheen M, Eger K and Steinfelder H J 2003 Biochem. Pharmacol. 65 603

8. Binaglow J B, Varnes M E, Roizer-Towle L, Clark E P, Epp E R, Astor M B and Hall E J 1986 Biochem. Pharmacol. 3577

9. Ames J R, Ryan M D and Kovacevic P 1986 Free Radical Biol. Med. 2277

10. Wardman P and Clarke E D 1976 Biochem. Biophys. Res. Commun. 69942

11. Hodgkiss J, Begg A C, Middleton R W, Parrick J, Stratford M, Wardman P and Wilson G 1991 Biochem. Pharmacol. 41553

12. Edwards D I 1986 Biochem. Pharmacol. 3553

13. Mester B, Claramunt R M, Elgreero J E, Atienza J, Barrio A G and Escario J A 1991 Chem. Pharm. Bull. 391990

14. Nunez-Vergara L J, Bollo S, Alvarez A, Blazquez M and Squella J A 1993 J. Electroanal. Chem. 345129
15. Nunez-Vergara L J, Garcia F, Dominguez M, La Fuente J De and Squella J A 1995 J. Electroanal. Chem. 381215

16. Masui M and Sayo H, 1956 Pharm. Bull. (Tokyo) 4 332

17. Squella J A, Sturm J C, Weiss-Lopez B, Bonta M and Nunez-Vergara L J 1999 J. Electroanal. Chem. 46690

18. Muller T E (ed.) 1971 Houben-Weyl Methoden der Organische Chemie 10th edn (Stuttgart: Georg Thieme Verlag)

19. Vogel's text book of practical organic chemistry 1996 (Essex: Addison Wesley Longman)

20. Lindquist J 1973 Anal. Chem. 451006

21. Atuma S S and Lindquist J 1973 Analyst 98886

22. Gosser K 1994 Cyclic voltammetry: Simulation and analysis of reaction mechanism (New York: $\mathrm{VCH}$ )

23. Symons T, Tocher J H, Tocher D H and Edwards D I 1991 Free Radicals Res. Commun. 1433

24. Tocher J H and Edwards D I 1992 Free Radical Res. Commun. 1619

25. Nunez-Vergara L J, Aldunate J, Letelier M E, Bollo S, Repetto Y, Morello A, Spencer P L and Squella J A 1995 Bioelectrochem. Bioenergy 38355

26. Squella J A, Letelier M E, Lindermeyer L and NunezVergara L J 1996 Chem. Bio. Interact. 99227

27. Vachalkova A, Novotny L and Blesova M 1996 Neoplasma 432

28. Rice M, Galus Z and Adams R N 1983 J. Electroanal. Chem. 14389

29. Squella J A, Bollo S and Nunez-Vergara L J 2005 Curr. Org. Chem. 9565

30. Amatore C, Saveant J M and Tessier D 1983 J. Electroanal. Chem. 14637 